\title{
Erratum to: Infusion of growth hormone into the hippocampus induces molecular and behavioral responses in mice
}

\author{
Paitoon Srimontri · Haruna Hirota $\cdot$ Hiroki Kanno • \\ Toshiya Okada $\cdot$ Yoshio Hirabayashi $\cdot$ Keiko Kato
}

Published online: 26 September 2014

(C) Springer-Verlag Berlin Heidelberg 2014

Erratum to: Exp Brain Res (2014) 232:2957-2966

DOI 10.1007/s00221-014-3977-y

The affiliations of Dr. Toshiya Okada and Dr. Yoshio Hirabayashi have been incorrectly published in the original publication. The correct affiliations are given below.

Paitoon Srimontri, Haruna Hirota, Keiko Kato: Faculty of Life Sciences, Kyoto Sangyo University, Motoyama, Kamigamo, Kita-ku, Kyoto 603-8555, Japan
Hiroki Kanno: Kobe City Meat Safety Inspection Office, 7-1-20, Karumo-dori, Nagata-ku, Kobe 653-0032, Japan

Toshiya Okada: Department of Integrated Structural Biosciences, Division of Veterinary Science, Graduate School of Life and Environmental Sciences, Osaka Prefecture University, Izumi-sano, Osaka 598-8531, Japan

Yoshio Hirabayashi: Laboratory for Molecular Membrane Neuroscience, Brain Science Institute, RIKEN, Wako, Saitama 351-0198, Japan

The online version of the original article can be found under doi:10.1007/s00221-014-3977-y.

P. Srimontri $\cdot$ H. Hirota $\cdot$ K. Kato $(\square)$

Faculty of Life Sciences, Kyoto Sangyo University,

Motoyama, Kamigamo, Kita-ku, Kyoto 603-8555, Japan

e-mail: kato@cc.kyoto-su.ac.jp

H. Kanno

Kobe City Meat Safety Inspection Office, 7-1-20,

Karumo-dori, Nagata-ku, Kobe, Japan

T. Okada

Division of Veterinary Science, Department of Integrated Structural Biosciences, Graduate School of Life and Environmental Sciences, Osaka Prefecture University, Izumi-sano, Osaka 598-8531, Japan

Y. Hirabayashi

Laboratory for Molecular Membrane Neuroscience, Brain

Science Institute, RIKEN, Wako, Saitama 351-0198, Japan 